\title{
From the Editor of Sexuality and Disability: International Communication: A Global Dimension to the Discussion of Sexuality and Disability
}

\author{
Sigmund Hough ${ }^{1}$
}

Published online: 8 May 2018

(C) Springer Science+Business Media, LLC, part of Springer Nature 2018

The journal of Sexuality and Disability remains a professional home and foundation for many, and a place of professional rejuvenation and exploration for still more. If you are new to the journal, we give to you a "BIG WELCOME" to participate as a reader, author, academician, clinician, educator, service provider, researcher, advocate, and individual or individuals seeking and sharing information. Over decades of contribution to the literature on sexuality and disability, we have been a part of the growth and understanding. Now most importantly, we continue to be a part of change in how we look and examine the topic, the need, and the response in terms of best practice- evidence based approaches. With the intelligence, experience, motivation and supportive commitment from our authors, readership, peer reviewers and editorial board, and resources with guidance from the Springer Staff, our journal's mission continues to be strong and meaningful. This international team effort continues as a strength in the field of sexuality and disability.

The sharing of international information, findings and perspectives allows one to move from the familiar and known, to increased awareness of differences and similarities. From what may be more standardized in opinion, belief and thought, to one of attentiveness to being exposed to new cultures, societies, choices, viewpoints and challenges. Being mindful highlights learning and growth. We continue to be mindful as we continue the journey.

Sexuality and Disability continues to provide original impact articles addressing the mental health and medical aspects of sexuality in relation to rehabilitation, hospital, academic and community settings, publishing up-to-date articles, case studies, clinical practice reports, reviews, featured articles, historical articles, special grand rounds topics, brief research reports and survey data reports. Value benefit is provided to authors through worldwide electronic exposure and professional access, while readership gains from scholarly contributions to advance the field through research, best-practice and educational articles. The refined lens of individual contributions from the local, national and international community continues to deliver a wealth of information on the topic of sexuality and disability for the reader. Thank you for joining our professional community.

Sigmund Hough

Sigmund_Hough@hms.harvard.edu

1 Beverly, MA, USA 